\title{
Can We Improve Patient Adherence by Harnessing Social Forces?
}

\author{
Ranak Trivedi, PhD ${ }^{1,2}$ and Steven M. Asch, MD, MPH ${ }^{1,3}$
}

${ }^{1}$ Center for Innovation to Implementation, VA Palo Alto Health Care System, Palo Alto, CA, USA; ${ }^{2}$ Department of Psychiatry and Behavioral Sciences, Stanford University, Stanford, CA, USA; ${ }^{3}$ Department of General Internal Medicine, Stanford University, Stanford, CA, USA.

J Gen Intern Med 34(6):785-6

DOI: $10.1007 / \mathrm{s} 11606-019-04856-4$

(c) Society of General Internal Medicine (This is a U.S. government work and not under copyright protection in the U.S.; foreign copyright protection may apply) 2019

$\mathrm{M}$ uch of the management of chronic illnesses occurs outside of the health care system. Selfmanagement-i.e., patient's ability to manage his or her treatment, lifestyle changes, and psychosocial effects of an illness - improves patients' quality of life and overall prognosis. It is common for patients and their family members or friends to partner around pharmacological and nonpharmacological tasks, a partnership that is generally beneficial to patients. Recent estimates indicate that $50-75 \%$ of patients with HF or diabetes have a family member who is regularly involved in their health care, ${ }^{1}$ and patients with low health literacy and greater illness burden are more likely to involve a family member. ${ }^{2}$

As noted by Lauffenburger et al. ${ }^{3}$ in this issue, half of all patients report medication nonadherence. There is a vast literature on patient-focused strategies to improve medication adherence, including improving knowledge, reducing forgetfulness, patient activation, self-efficacy, motivation, and increasing proximal rewards via incentives. However, patients manage their health within the context of their social network and patients' high perceived social support is associated with better outcomes. Family members and friends may provide practical support (e.g., prepare meals, take patient to appointments) and emotional support to buoy patients' mood, encourage them to follow medical recommendations, and reward them for their efforts. Involving social networks can improve patients' quality of life, self-efficacy, and relationship quality ${ }^{4}$ and reduce hospitalizations. ${ }^{5}$

It is with this thesis - that family members and friends play key roles in medication adherence - that Lauffernburger et al. ${ }^{3}$ examined insurance data to see if family members' medication adherence predicted patients' medication adherence within 12 months. They examined adherence among 254,144 patients being treated for diabetes, hypertension, hyperlipidemia, hypothyroidism, or mental health conditions (i.e., depression, anxiety), and identified their family members using linked data. Family members' medication adherence was identified

Published online March 18, 2019 within the 1 year preceding the patients' date of study entry. Pharmacy refill data were used to calculate medication adherence for both patients and their family members. Patients whose family members were fully adherent $(>80 \%)$ had higher adherence than those whose family members were not fully adherent. Rates of full adherence among patients whose family members were fully adherent were $37.3 \%$, compared to $26.9 \%$ if family members were not fully adherent. The relative risk was stronger if both patients and family members used cardiometabolic medications (aRR, 1.35; 95\% $\mathrm{CI}=1.28-1.31$ ). If both patients and family members were treated for the same condition, $38 \%$ of patients were fully adherent.

The authors are to be applauded for finding an innovative way to ascertain that behaviors of family members and patients are connected. Such methodological advances are critical because current electronic health records either do not capture the role of family, or do so superficially through marital status or noting next of kin. Strengths of their study also include the sample size, and the advanced statistical methods to limit confounds of time-varying elements. Having comprehensive data from both patients and family members can lead to applications of advanced statistical methods such as structural equation modeling, the Common Fate Model, and the Actor-Partner Interdependence Model. Preliminary applications of these models shed light on the reciprocal emotional experiences and well-being of patients and their family members. ${ }^{6}$ Social network analysis has also shown that healthy and unhealthy behaviors are contagious within social networks, and this study bolsters existing evidence using a large, administrative dataset. Developing innovative ways to use existing datasets will deepen our understanding of the linkages between the behaviors of patients and their family members.

The authors speculate that encouragement and reinforcement are the most likely explanations of their findings. However, family relationships may be toxic, and negative interactions marked by criticism may worsen adherence and clinical outcomes. ${ }^{8}$ Both social approval and disapproval are powerful forces that affect human behavior, and maintaining healthy behaviors is easier when it is congruent with the values or expectations within the social context. Behavioral contagion theory has recently highlighted that both healthy and unhealthy behaviors can spread within social networks. The 
widely cited example of social contagion is the rise of obesity due to the widespread conformity to mindless eating, ${ }^{7}$ even though re-analyses of these data contradicted the study conclusions. Future investigations could make an important contribution by providing a holistic picture of the positive and negative effects of social forces.

Two other explanations warrant discussion: affordability and knowledge. Affordability is one of the most common barriers to medication adherence which affects families rather than single individuals. In Lauffenburger et al. study, all the participants were insured and had benefits that covered the disease conditions that were studied. It is possible that the concordant adherence within families was at least in part due to shared affordability. The lack of knowledge is also a common barrier to adherence and forms the cornerstone of selfmanagement programs and education. Family members who understand the importance of adherence, and/or are adherent themselves, may be role models and provide positive reinforcement to patients via encouragement. Specific to this study, patients prescribed medications later than the family members may benefit from family members earlier experiences, as family members may have learned to avoid common pitfalls of nonadherence (e.g., forgetting).

Study findings should be considered within the burgeoning literature on the role of informal caregivers (i.e., family members and friends who directly help patients with their illness) in supporting medication and nonmedication adherence. The authors erroneously note interventional studies of social support are limited to HIV and other communicable diseases. Rather, the role of informal caregivers (family members or friends) has been well -elucidated among adult patients with noncommunicable diseases, most notably dementia, but also cancer, cardiovascular disease, diabetes, and depression. Empirically tested interventions engage informal caregivers at all levels of care, including providing them training and knowledge regarding patient care and interventions to reduce caregiver stress. Technology-enabled tools are also well-suited to engaging caregivers, as evidenced by interventions across depression, heart failure, and diabetes. ${ }^{9}$

A relatively new and exciting area is developing interventions that improve the family functioning around a patient. Since family members and friends form the scaffolding of patient care, patients are often only as resilient as the family unit. The Couples' Coping Enhancement Training program was developed to improve relationship quality among couples coping with a chronic illness and has led to similar programs in cancer (Partners in Coping Program) and other adaptations. Our SUCCEED (Self-Care Using Collaborative Coping Engagement in Diseases) program is a family-centered self-management program intended to reduce individual and relationship stress, and improve communication and collaboration around self-management. ${ }^{10}$ Such interventions are timely as major health care systems are exploring strategies to enable patients to age in place and prevent institutionalizations.

The modernization of medicine has become disconnected from patients, but also the social ties that have bonded us together across millennia. System-wide changes may be necessary to shift from a patient-centered health care system to a family-centered health care system. Understanding and improving the engagement of family members, and supporting them in their roles as caregivers, are crucial next steps to enhance not only patient well-being, but the well-being of our societies as a whole.

Corresponding Author: Ranak Trivedi, $\mathrm{PhD}$; Center for Innovation to Implementation, VA Palo Alto Health Care System, Palo Alto, CA, USA (e-mail: ranakt@stanford.edu).

\section{Compliance with Ethical Standards:}

Conflicts of Interest: The authors declare that they do not have a conflict of interest.

\section{REFERENCES}

1. Rosland AM, Heisler M, Choi HJ, Silveira MJ, Piette JD. Family influences on self-management among functionally independent adults with diabetes or heart failure: do family members hinder as much as they help?, Chronic illness. 2010;6(1):22-33.

2. Rosland AM, Piette JD, Choi H, Heisler M. Family and friend participation in primary care visits of patients with diabetes or heart failure: patient and physician determinants and experiences. Med Care. 2011;49(1):37-45.

3. Lauffenburger JC, Khan NF, Brill G, Choudhry NK. Quantifying social reinforcement among family members on adherence to medications for chronic conditions: A US-based retrospective cohort study. J Gen Intern Med. DOI: https://doi.org/10.1007/s11606-018-4654-9.

4. Martire LM, Schulz R, Keefe FJ, Rudy TE, Starz TW. Couple-oriented education and support intervention for osteoarthritis: Effects on spouses' support and responses to patient pain. Families, Systems, \& Health. 2008;26(2): 185-195.

5. Nichols LO, Martindale-Adams J, Burns R, Graney MJ, Zuber J. Translation of a dementia caregiver support program in a health care system-REACH VA. Arch Intern Med. 2011;171(4):353-359.

6. Dorros SM, Card NA, Segrin C, Badger TA. Interdependence in women with breast cancer and their partners: an interindividual model of distress. Journal of consulting and clinical psychology. 2010;78(1):121-125.

7. Christakis NA, Fowler JH. The spread of obesity in a large social network over 32 years. $N$ Engl J Med. 2007;357(4):370-379.

8. Vitaliano PP, Young HM, Russo J, Romano J, Magana-Amato A. Does expressed emotion in spouses predict subsequent problems among care recipients with Alzheimer's disease? J Gerontol. 1993;48(4):P202-209.

9. Piette JD, Striplin D, Marinec N, et al. A Mobile Health Intervention Supporting Heart Failure Patients and Their Informal Caregivers: A Randomized Comparative Effectiveness Trial. Journal of medical Internet research. 2015;17(6):e142.

Publisher's Note: Springer Nature remains neutral with regard to jurisdictional claims in published maps and institutional affiliations. 